\title{
A Fully Integrated Real-Time Detection, Diagnosis, and Control of Community Diarrheal Disease Clusters and Outbreaks (the INTEGRATE Project): Protocol for an Enhanced Surveillance System
}

Kirsty Marie McIntyre ${ }^{1,2}, \mathrm{BSc}, \mathrm{PhD}^{\dagger}$; Frederick J Bolton ${ }^{1}$, PhD; Rob M Christley ${ }^{1}$, PhD; Paul Cleary ${ }^{2,3}, \mathrm{MD}$, PhD; Elizabeth Deja ${ }^{4}, \mathrm{PhD}$; Ann E Durie ${ }^{1}$, MSc; Peter J Diggle ${ }^{2,5}$, PhD; Dyfrig A Hughes ${ }^{6}, \mathrm{PhD}$; Simon de Lusignan ${ }^{7,8}$, MD; Lois Orton ${ }^{4}$, PhD; Alan D Radford ${ }^{9}$, PhD; Alex J Elliot ${ }^{10}$, PhD; Gillian E Smith ${ }^{10}$, MD; Darlene A Snape ${ }^{4}$, PhD; Debbi Stanistreet ${ }^{4}, \mathrm{PhD}$; Roberto Vivancos ${ }^{3}, \mathrm{MD}, \mathrm{PhD}$; Craig Winstanley ${ }^{11}$, PhD; Sarah J O’Brien ${ }^{2,4}$, MD

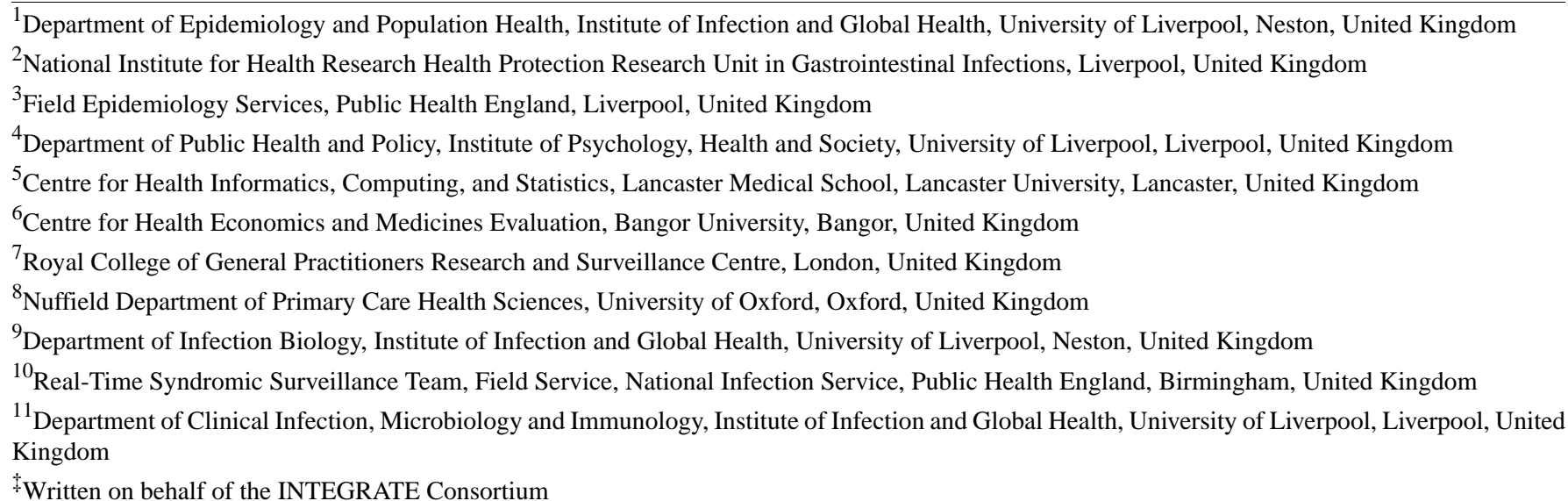

\section{Corresponding Author:}

Kirsty Marie McIntyre, BSc, PhD

Department of Epidemiology and Population Health

Institute of Infection and Global Health

University of Liverpool

Chester High Road

Neston, CH64 7TE

United Kingdom

Phone: 441519758307

Email: k.m.mcintyre@ liverpool.ac.uk

\begin{abstract}
Background: Diarrheal disease, which affects 1 in 4 people in the United Kingdom annually, is the most common cause of outbreaks in community and health care settings. Traditional surveillance methods tend to detect point-source outbreaks of diarrhea and vomiting; they are less effective at identifying low-level and intermittent food supply contamination. Furthermore, it can take up to 9 weeks for infections to be confirmed, reducing slow-burn outbreak recognition, potentially impacting hundreds or thousands of people over wide geographical areas. There is a need to address fundamental problems in traditional diarrheal disease surveillance because of underreporting and subsequent unconfirmed infection by patients and general practitioners (GPs); varying submission practices and selective testing of samples in laboratories; limitations in traditional microbiological diagnostics, meaning that the timeliness of sample testing and etiology of most cases remains unknown; and poorly integrated human and animal surveillance systems, meaning that identification of zoonoses is delayed or missed.
\end{abstract}

Objective: This study aims to detect anomalous patterns in the incidence of gastrointestinal disease in the (human) community; to target sampling; to test traditional diagnostic methods against rapid, modern, and sensitive molecular and genomic microbiology 
methods that identify and characterize responsible pathogens rapidly and more completely; and to determine the cost-effectiveness of rapid, modern, sensitive molecular and genomic microbiology methods.

Methods: Syndromic surveillance will be used to aid identification of anomalous patterns in microbiological events based on temporal associations, demographic similarities among patients and animals, and changes in trends in acute gastroenteritis cases using a point process statistical model. Stool samples will be obtained from patients' consulting GPs, to improve the timeliness of cluster detection and characterize the pathogens responsible, allowing health protection professionals to investigate and control outbreaks quickly, limiting their size and impact. The cost-effectiveness of the proposed system will be examined using formal cost-utility analysis to inform decisions on national implementation.

Results: The project commenced on April 1, 2013. Favorable approval was obtained from the Research Ethics Committee on June 15, 2015, and the first patient was recruited on October 13, 2015, with 1407 patients recruited and samples processed using traditional laboratory techniques as of March 2017.

Conclusions: The overall aim of this study is to create a new One Health paradigm for detecting and investigating diarrhea and vomiting in the community in near-real time, shifting from passive human surveillance and management of laboratory-confirmed infection toward an integrated, interdisciplinary enhanced surveillance system including management of people with symptoms.

International Registered Report Identifier (IRRID)： DERR1-10.2196/13941

(JMIR Res Protoc 2019;8(9):e13941) doi: 10.2196/13941

\section{KEYWORDS}

gastrointestinal diseases; syndromic surveillance; microbiology; diarrhea; vomiting

\section{Introduction}

\section{Background}

Diarrheal disease, affecting 1 in 4 people in the United Kingdom annually [1], is the most common cause of infectious disease outbreaks in community and health care settings. Traditional surveillance methods tend to detect point-source outbreaks of diarrhea and vomiting; however, they are less effective at identifying low-level and intermittent contamination of the food supply, unless the organism is very rare. Furthermore, it may take up to 9 weeks for infections to be confirmed by a reference laboratory, reducing recognition of slow-burn outbreaks that can affect hundreds or thousands of people over a wide geographical area.

There is a need to address fundamental problems inherent in traditional surveillance for diarrheal disease. First, surveillance depends on the examination of stool samples obtained from symptomatic patients attending their general practitioner (GP). The submission practices and selective testing of samples in laboratories can vary and potentially fragment current laboratory-based surveillance systems. Furthermore, as fewer people present in person to their GP, laboratory-based systems have become less sensitive; the hidden burden of disease has increased [1]. Second, limitations of traditional microbiological diagnostic methods mean that the etiology of diarrhea in most cases remains unknown. Third, diagnostics are conducted in a hierarchical manner (local detection, confirmation, and typing centrally at national reference laboratories), which can take several days and can be delayed during busy periods such as when an outbreak investigation is underway. Finally, although many diarrheal diseases are zoonotic, human and animal surveillance systems are poorly integrated, meaning that identification of zoonotic events, including emergence of new or antibiotic-resistant strains, is delayed or missed altogether.

\section{Overall Study Aim}

The overall aim of this study is to create a new One Health paradigm for detecting and investigating diarrhea and vomiting in the community in near-real time, shifting from passive human surveillance for gastrointestinal (GI) illness and management of laboratory-confirmed infection toward an integrated, interdisciplinary enhanced surveillance system including management of people with symptoms.

\section{Contribution to the Field}

The comparison of the use of syndromic surveillance for cluster detection and targeted sampling within the community with the use of traditional surveillance will provide a series of improvements to the surveillance of GI disease. We hypothesize that enhanced GI surveillance will allow the following:

- Faster identification of outbreaks of GI disease

- More accurate characterization of the hidden burden of disease (underreporting of episodes of illness in which patients do not visit GPs in person). This will result in an observed increase in the incidence of outbreaks

- Identification of a greater number of routes for transmission of pathogens that cause GI illness.

The integration of human and animal syndromic surveillance systems and the use of modern microbiological methods within this project are hypothesized to facilitate (1) faster detection of zoonotic transmission events; (2) earlier identification of a greater spectrum of disease-transmitting pathogens, reducing the diagnostic gap for GI disease; and (3) a reduction in the numbers of false-positive and false-negative stool samples.

There will be differences in the costs and benefits of using improved surveillance methods to detect outbreaks of GI disease earlier compared with using traditional surveillance methods. Potentially, these differences could be in parameters relating to host-pathogen interactions; rate parameters that define the transition of patients among relevant states (eg, susceptible, 
diseased, and symptomatic and/or infectious); test characteristics, defined by sensitivity, specificity, and positive and negative predictive values; costs (associated with screening, patients' use of National Health Service [NHS] community, primary and secondary care services, treatments, and other investigations); health outcomes (defined by health state utilities); personal social services; days absent from work or education; and other potential cost impacts.

\section{Overall Objectives of the Research Program}

The overall objectives of this research program are to (1) develop and implement new sampling and microbiological testing algorithms, including strategies for pathogen discovery and evolutionary biology; (2) run the new system alongside the existing system to assess its performance against a set of outcome-based indicators including time to detection of event, compliance with sampling among people with symptoms, numbers of false-positive and false-negative stool samples, and diagnostic yield; and (3) determine the costs and benefits of the new system.

\section{Methods}

\section{Setting}

The setting is the North West area of England (population 7.1 million).

\section{Case Recruitment and Informed Consent}

A total of 4 data streams will feed in real time into the new surveillance program. They are NHS 111 telephone triage data on symptoms of vomiting and diarrhea (a real-time syndromic surveillance system operated by Public Health England [PHE]), data from the Small Animal Veterinary Surveillance Network [2], and Salmonella data from the Animal and Plant Health Agency (APHA).

The fourth data stream will be derived from general practices in the Royal College of General Practitioners' Research and Surveillance Centre National Monitoring Network (RCGP RSC NMN) [3]. Members of the public with symptoms of acute gastroenteritis including a case definition of vomiting and diarrhea who seek health advice from general practices in the RCGP RSC NMN will be invited to submit a stool sample for microbiological examination. Their consent for this procedure will be sought because normal care would not necessarily entail stool sampling for most patients unless their symptoms were severe or had persisted for a long time. It is possible that most patients will be recruited as part of a telephone consultation with a member of their primary health care team (physician assessment by telephone). The primary health care team will arrange for the patient to receive through the post an invitation letter, an information sheet about the study, a consent form, a stool sampling kit with a reply-paid envelope, and a short Public Health Acute Gastroenteritis questionnaire (which is part of routine public health practice) with a reply-paid envelope. Patients who present at a general practice in the RCGP RSC NMN will receive these items in person. Patients who consent to take part and provide a stool sample will be recruited into the study. Consent statements agreed to in the study consent form include acknowledgment that taking part in the study is voluntary and that consenting patients can leave at any time. Figures 1 and 2 describe the study recruitment procedure, processes, and data flows using flow diagrams.

We aim to recruit 6000 participants. This will allow us to detect the period (annual) prevalence of symptomatic GI infection in the community of $20 \% \pm 1 \%$.

\section{Sample Processing}

On receipt at 1 of the 3 diagnostic laboratories taking part (Royal Liverpool and Broadgreen University Hospitals NHS Trust, Central Manchester University Hospitals NHS Foundation Trust, or Lancashire Teaching Hospitals NHS Foundation Trust), the stool sample will be divided into 2 parts. One part of the sample will be processed according to routine clinical practice at each of the 3 laboratories. The other half will be processed with a rapid first-line diagnostic screen, using a molecular multiplex real-time polymerase chain reaction (PCR) assay (a commercially available CE-marked kit [Luminex xTAG Gastrointestinal Pathogen Panel, xTAG GPP] [4]), which incorporates most of the major community GI pathogens relevant to the United Kingdom. This will be complemented by tests for Enteroaggregative Escherichia coli and Sapovirus, which have been incorporated into the xTAG GPP assay, using assays already developed for PHE's Olympics Response [5]. Downstream from the rapid xTAG GPP diagnosis, an algorithm for testing stool samples from presumed outbreaks using next-generation sequencing technologies will allow for molecular characterization of known pathogens. Where a pathogen has not been identified (likely in $60 \%$ of samples), samples from clinically severe outbreaks will be fast-tracked and characterized using a relatively low-throughput platform for combined RNA/DNA viromes and bacterial metagenomes. If they are from less clinically urgent cases, they will be sequenced at reduced cost on a high-throughput platform.

If a patient does not wish to take part in the study but does submit a stool sample, this case will be processed according to routine clinical practice.

All results will be reported to the patient's GP. Results from the Luminex assays will be issued as an interim report through Telepath, which is the routine electronic reporting system between diagnostic laboratories and general practice, and the results of the routine clinical practice assays will be issued as a final report through Telepath. In each laboratory, an experienced consultant medical microbiologist, who is a coinvestigator on the grant with research time costed into it, will be available to discuss any discrepant results with the patient's GP. The most likely scenario is that a stool sample that provides negative results using traditional methods will provide positive results using the xTAG GPP. This is to be expected because the XTAG GPP, which is a multiplexed PCR, will detect the presence of pathogen DNA/RNA in stool even if an organism has died-off in the sample during transit to the laboratory. 
Figure 1. Patient recruitment flow diagram and study processes for the INTEGRATE project. ASAP: as soon as possible; xTAG GPP: Luminex xTAG Gastrointestinal Pathogen Panel.

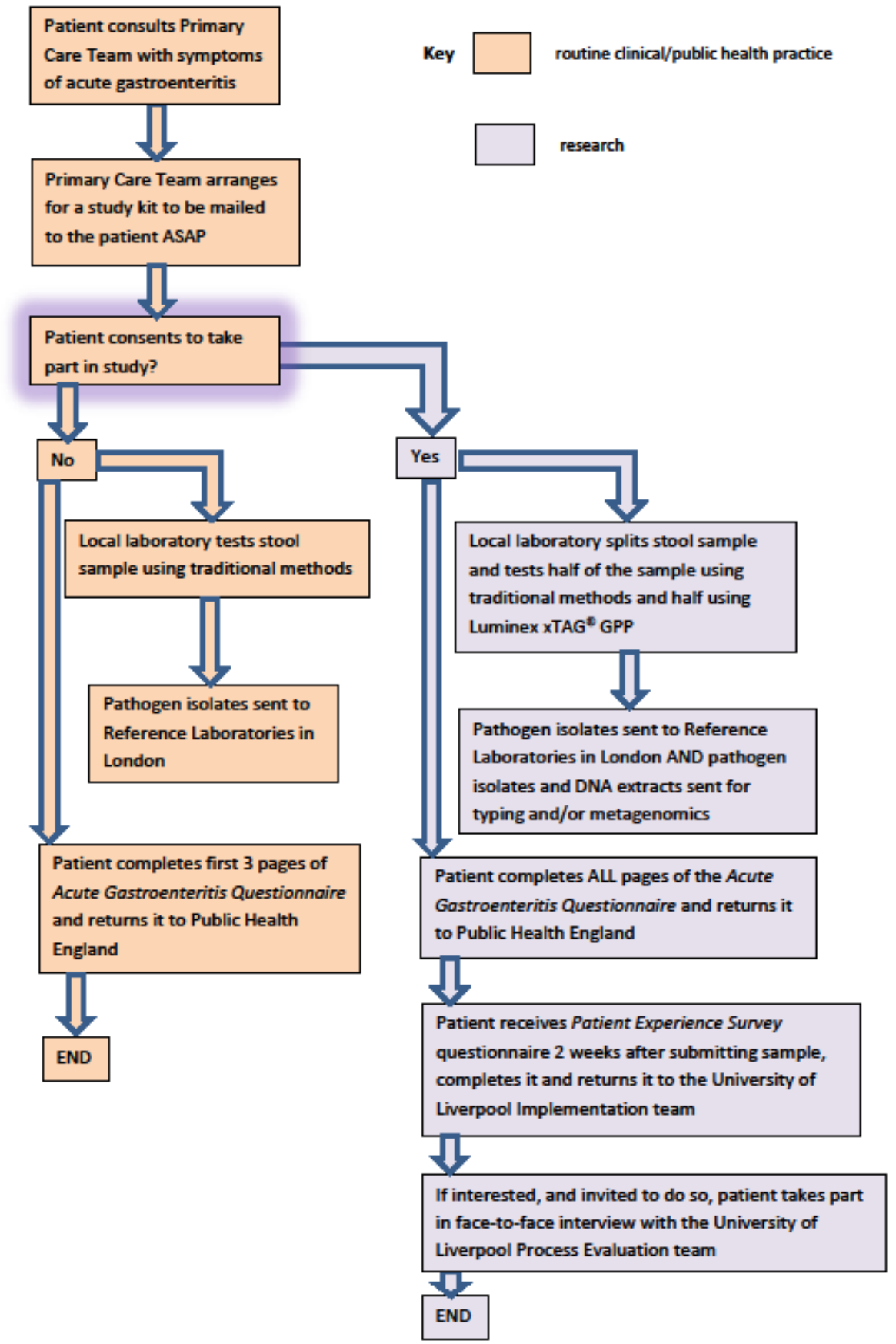


Figure 2. Post patient recruitment data flow for the INTEGRATE project. AEGISS: Ascertainment and Enhancement of Gastrointestinal Surveillance and Statistics; AGE: Public Health Acute Gastroenteritis; HES: Hospital Episode Statistics; HSCIC: Health and Social Care Information Centre; NHS: National Health Service; PHE: Public Health England; RCGP: Royal College of General Practitioners; UoL: University of Liverpool.

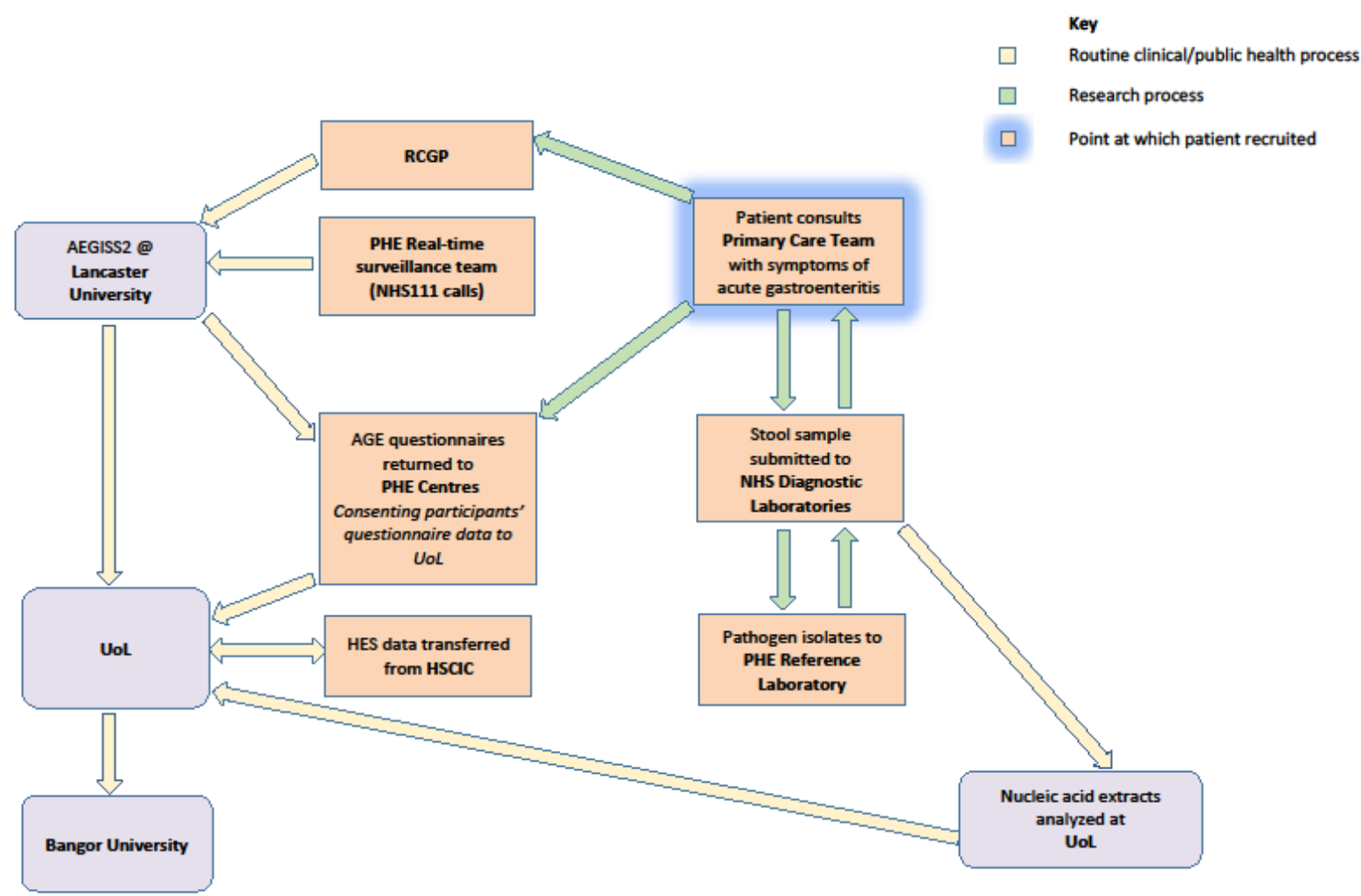

\section{Public Health Acute Gastroenteritis Questionnaires}

Public Health Acute Gastroenteritis (AGE) questionnaires will be returned to PHE's health protection teams using reply-paid envelopes. These questionnaires contain the routine follow-up information that is collected and collated by the PHE to assist outbreak detection or investigation. In addition, these questionnaires will contain questions on quality of life during the acute illness. Quality of life will be measured using the EuroQol-5D-3L [6], which is the gold standard tool. This gives snapshots of quality of life at points in time. At this time point (time point 1 ), we will capture the quality of life during the acute illness.

\section{Resource Use and Costs}

Resource use and costs will be assessed from several different perspectives, described below, including the public sector and patients.

\section{Public Sector, Including National Health Service and Social Services}

Consistent with National Institute for Health and Care Excellence's (NICE) methods for the development of public health guidance, we will adopt a public sector costing perspective. Although productivity costs are not routinely included in analyses, we will collect the data for consideration in sensitivity analysis. Unit costs will be derived from standard sources such as the Personal Social Services Research Unit
Costs of Health and Social Care, the British National Formulary for drug costs, and NHS reference costs. The costs of laboratory and public health services will be obtained from PHE.

\section{Patients}

Patients who indicate in their consent form that they are willing to be contacted about a Patient Experience Survey (PES) will receive a questionnaire that seeks information about resource use 2 weeks after returning their stool sample pot. Most patients will have recovered fully 2 weeks later, and therefore, a complete picture of the costs they incurred because of their illness should be available. The short resource use questionnaires will capture details about the use of health care services, personal social services, days absent from work or school, and other potential costs. Quality of life, using EuroQol-5D-3L, will be assessed during the acute episode (time point 1 ) and at 2 weeks post recovery (time point 2). It will be used to calculate quality-adjusted life-years (QALYs).

\section{Hospital Episode Statistics}

Patients' use of secondary care services will be assessed by accessing Hospital Episode Statistics data sourced from NHS Digital. This will require a bespoke download, matching patients who have consented according to their NHS number, name, and date of birth, following a standard operating procedure to ensure patient anonymity and data protection. 


\section{Costs of Technology}

These will include setup costs, including capital costs, the marginal costs of delivering the service, and the cost implications for patients using the NHS services. These costs will be obtained from Luminex and from time and motion studies conducted at participating microbiology laboratories.

\section{Patient Experience Survey}

The purpose of this survey is to explore service users' and, where relevant, their caregivers' perceptions and experiences of accessing the INTEGRATE service. Questions contained within the PES were developed from themes identified from the extant literature and include (1) motivations for accessing health care in relation to diarrhea; (2) predisposing or enabling motivations for accessing a given health care service; (3) experience and perceptions including the acceptability of self-stool sampling; and (4) dimensions of patient satisfaction, including, for example, communication and clarity of information.

Members of the public eligible to participate in the survey will be either a patient (aged $\geq 16$ years) who has recently suffered from diarrhea and provided a stool sample, or a person who has parental, guardian, or caregiver responsibility for, and who will act as a proxy on behalf of, a patient who has recently suffered from diarrhea and provided a stool sample.

There was no upper age limit for sampling patients. However, where the patient is a minor (aged $<16$ years), the parent, guardian, or caregiver will be invited to respond on their behalf (as a proxy). We estimate approximately 5000 members of the public will be eligible to take part in the survey within a time frame of 15 months. During this period, all eligible participants will be consecutively recruited to participate in the survey as a means to achieving maximum variation in clinical and sociodemographic characteristics and to facilitate comparison across key purposive sampling criteria [7]. Participants will be invited to complete the questionnaire 2 weeks after returning their stool self-sample pot, allowing a 3-week recall period from the date of contact with the NHS.

\section{Survey Distribution}

When a member of the public contacts a collaborating GP, they will receive information in their study pack about the patient experience survey. If they express an interest in finding out more about this survey, they will be asked to identify a preferred contact route by either receiving a self-completion questionnaire through the post or accessing a Web-based self-completion questionnaire.

If prospective participants choose to receive this information through the post, they will be sent a public survey information pack containing an introductory letter, an information sheet explaining the aims of the study and the processes involved in participating in the survey, a copy of the self-completion questionnaire, and a prepaid return envelope addressed to the Project Office at the University of Liverpool. If prospective participants prefer to access these details online, they will receive an email. The email will include an electronic link to a secure, Web-enabled system containing a study introduction page. If interested, prospective participants can then access 2 further links. The first link will open an information page, which will outline the aims of the study and processes involved in participating in the online survey. The second link will enable prospective participants to access and complete the electronic version of the self-completion questionnaire (through the PHE Select Survey facility [8]).

Participants will be given 2 weeks to complete and return/submit their questionnaire. Nonresponders will be prompted (through their preferred route) with 1 follow-up reminder. These will include, as appropriate, a follow-up letter containing a further copy of the questionnaire and a prepaid return envelope or a follow-up email with an electronic link to the online questionnaire. The use of reminders is generally endorsed in texts on survey methods [9].

\section{Summary of Outcome Measures}

The outcome measures to be quantified within the research project are summarized in Table 1 .

\section{Plan of Analyses}

\section{Anomaly Detection}

The underlying statistical model for human case incidence will be a spatiotemporal Cox process [10] in which the rate of calls at location $x$ and time $t$ is modeled as $\rho(x, t)=\lambda(x) \mu(t) R(x, t)$, where $\lambda(x)$ and $\mu(t)$ describe the normal patterns of variation in the spatial and temporal dimensions, respectively, of the call rate, thereby taking account of the geographical distribution of the user population, seasonal variation in disease risk, and reporting artifacts such as day-of-the-week effects. The term $R(x, t)$ is a stochastic process with expected value 1 and represents unforeseen, spatially and temporally localized variations in the underlying disease risk.

Model parameters will be estimated by likelihood-based methods and fed into algorithms that update the predictive distribution of the unexpected component $R(x, t)$ automatically on receipt of each day's incident call data. Results will be posted overnight in the form of maps showing localities, if any, where the data indicate a high probability that the current value of $R(x, t)$ exceeds a specified threshold.

\section{System Performance}

The performance of the new surveillance system compared with routine sampling will be assessed by analyzing the outcome-based indicators and comparing time with detection, decision to act, and the size of outbreaks described using both traditional and new diagnostic systems (range, mean, and median number of cases), outbreak settings, modes of transmission identified, and vehicles identified. Each critical time point along the diagnostic and detection pathway from symptom onset to diagnosis and detection of a cluster or outbreak will be examined. 
Table 1. Resource use and costs outcome measures to be quantified within the research program.

\begin{tabular}{|c|c|}
\hline Outcome measures & How to measure \\
\hline \multicolumn{2}{|l|}{ Resource use and costs outcome measures } \\
\hline Use of health care services & Resource use questionnaire $\left(\mathrm{PES}^{\mathrm{a}}\right)$ to patients \\
\hline Use of personal social services & Resource use questionnaire (PES) to patients \\
\hline Days absent from work or education & Resource use questionnaire (PES) to patients \\
\hline Other potential cost impacts & Resource use questionnaire (PES) to patients \\
\hline Use of secondary care services & Hospital Episode Statistics from NHS ${ }^{\mathrm{b}}$ Digital \\
\hline Costs of technology & $\begin{array}{l}\text { Interviews with Luminex (new technology) and time and motion studies at microbiology } \\
\text { laboratories (existing technology) }\end{array}$ \\
\hline Health outcome & $\begin{array}{l}\text { EQ-5D-3L } \mathrm{L}^{\mathrm{c}} \text { questionnaire administered at } 2 \text { time points: time point } 1 \text {, during the acute } \\
\text { illness; and time point } 2,2 \text { weeks after return of the Acute Gastroenteritis Questionnaire }\end{array}$ \\
\hline \multicolumn{2}{|l|}{ System outcome measures ${ }^{d}$} \\
\hline Time to detection of event & $\begin{array}{l}\text { Laboratory records, date of AEGISS }{ }^{\mathrm{e}} \text { anomaly detection, date that Consultants in Com- } \\
\text { municable Disease Control initiate an investigation }\end{array}$ \\
\hline $\begin{array}{l}\text { Compliance with sampling among people with symp- } \\
\text { toms }\end{array}$ & $\begin{array}{l}\text { Laboratory records (number of samples requested) and } \mathrm{GP}^{\mathrm{f}} \text { records (number of samples } \\
\text { submitted) }\end{array}$ \\
\hline Time to detection of a positive result & Laboratory records \\
\hline $\begin{array}{l}\text { Numbers of false-positive and false-negative stool } \\
\text { samples }\end{array}$ & Laboratory records \\
\hline Positive predictive value & $\begin{array}{l}\text { Calculated from laboratory records using the formula: } \Sigma \text { true positives } / \Sigma \text { test outcome } \\
\text { positives (ie, true positives + false positives) }\end{array}$ \\
\hline Diagnostic gap & Laboratory records: percentage of negative samples using either system \\
\hline Size of outbreaks detected & Outbreak investigation reports: range, mean, and median numbers of cases \\
\hline
\end{tabular}

${ }^{\text {a} P E S: ~ P a t i e n t ~ E x p e r i e n c e ~ S u r v e y . ~}$

${ }^{b}$ NHS: National Health Service.

${ }^{c}$ EQ-5D-3L: EuroQol-5D-3L descriptive system.

${ }^{\mathrm{d}}$ These will be captured for traditional methods and new diagnostic technology.

eAEGISS: Ascertainment and Enhancement of Gastrointestinal Surveillance and Statistics.

${ }^{\mathrm{f}} \mathrm{GP}$ : general practitioner.

\section{Economic Modeling}

The model structure will be based on a decision analysis in which the alternative options will be specified according to treatment pathways and strategies for public health intervention. The impact and scale of outbreaks will be modeled using agent-based models in which hypothetical cohorts are subject to an instantaneous rate of infection, which varies depending on the proportion of the population who are infected. This approach has several advantages over the traditional health economic models, which are restrictive in their predictive capabilities, and scenario testing. The model will be parameterized with point estimates and associated variances, derived from a purposive review of the published literature, from routinely collected data from PHE (both historical and contemporary), and from data generated during the research. These will include parameters relating to host-pathogen interactions; rate parameters that define the transition of patients among relevant states (eg, susceptible, diseased, and symptomatic and/or infectious); test characteristics, defined by sensitivity, specificity, and positive and negative predictive values; costs (associated with screening, patients' use of NHS community, primary and secondary care services, treatments, and other investigations); and health outcomes (defined by health state utilities based on UK tariff scores assigned to each model state and mortality estimates).

\section{Calculating and Judging Cost-Effectiveness}

Expected costs and benefits will be estimated to calculate incremental cost-utility ratios (costs per QALY gained) for a range of scenarios, specified by infection type, clinical course, and public health response. Estimates of the incremental cost-effectiveness ratios (ICERs) will be compared with the $£ 20,000$ to $£ 30,000$ per QALY threshold of cost-effectiveness set by NICE, and a range of one-way sensitivity analyses will be conducted to assess the robustness of the analysis. These will be presented as a Tornado plot. Multivariate sensitivity analyses will be applied where interaction effects are suspected. The joint uncertainty in all parameter estimates will be propagated through the model by use of probabilistic sensitivity analysis and construction of cost-effectiveness acceptability curves that present the probability of clinical strategies being 
cost-effective, conditional on the chosen threshold for cost-effectiveness (representing the marginal value of health). Scenario analyses representing, for example, changes in service configuration will be conducted to estimate a range of ICERs for different circumstances.

\section{Ethics}

For the human case data, ethics permissions and approvals have been obtained from the following:

- National Research Ethics Service, REC reference: 15/NW/0233

- NHS Health Research Authority Confidential Advisory Group (CAG), CAG reference: 15/CAG/0131

- The Information Governance Toolkit, Department of Health and Social Care hosted by the Health and Social Care Information Centre (now NHS Digital), UoL reference: 8HN20, Lancaster University reference: EE133831-HAM-EAOPOCR

- NHS Research Management and Governance Committees, IRAS number: 173789

- University of Liverpool Ethics Sub-Committees, reference: UoL001111

- Honorary NHS contracts, research passports, and letters of access have been obtained for research staff working on the project as necessary.

\section{Results}

The project commenced on April 1, 2013. Favorable approval was obtained from the Research Ethics Committee on June 15, 2015, and the first patient was recruited on October 13, 2015, with 1407 patients recruited and samples processed using traditional laboratory techniques as of March 2017.

\section{Discussion}

This study investigates whether modern microbiological methods can be used to improve surveillance for GI disease while also examining the costs and limitations associated with the enhanced system. It compares the results obtained using traditional laboratory techniques with those obtained using modern sensitive molecular and genomic microbiology techniques. The strength of the study is the collaboration between lead public health partners and researchers in this field. However, there are a number of challenges in this study. For example, the plans for work are based on the assumption that implementation of the surveillance streams and their providers will continue in their current form for at least the period of study recruitment. Ethical permissions are granted under this proviso, but as with the provision of all health services, changes can occur rapidly. If there are changes to the study protocol, then these must be reflected in amendments to the ethics agreements, and the research governance including confidentiality agreements required for this to happen can take a significant amount of time to go through the review process, delaying the progression of data collection. Another challenge is that of recruiting a sufficiently large number of patients for analysis, to allow reasonable comparison of the results of the traditional and modern microbiological testing. This is particularly true, given that GPs often do not encourage patients to provide a stool sample unless they have had clinical GI symptoms for an extended period or unless they are in a high-risk group such as those who are young, old, or immunocompromised.

\section{Acknowledgments}

The authors acknowledge the Department of Health and Social Care and the Wellcome Trust for the funding received for this project through the Health Innovation Challenge Fund (grant reference: HICF-T5-354). The authors acknowledge the support of the University of Liverpool Sponsorship Review and Approval Committee, patients registered with RCGP RSC general practices as well as the practices that allowed their data to be shared, Apollo Medical Systems, EMIS, In-Practice Vision, and TPP for support and collaboration with data extraction. The authors would like to thank Simone Nudds, Gillian Byrne, and David Knight from Luminex Corporation for their support. Finally, the authors would also like to thank NHS 111 for providing permission to use the anonymized NHS 111 syndromic surveillance data. SdL has subsequently received funding through the University of Surrey to explore household transmission of acute gastroenteritis.

SJOB, PC, PJD, NH, CHF, and KMM are affiliated to the National Institute for Health Research Health Protection Research Unit (NIHR HPRU) in Gastrointestinal Infections at the University of Liverpool in partnership with PHE, in collaboration with University of East Anglia, University of Oxford, and the Quadram Institute. MB, RMC, SD, PJD, NH, KMM, ADR, and RV are affiliated to the NIHR HPRU in Emerging and Zoonotic Infections at the University of Liverpool in partnership with PHE, in collaboration with Liverpool School of Tropical Medicine. The views expressed are those of the author(s) and not necessarily those of the NHS, the NIHR, the Department of Health and Social Care or the PHE.

The authors thank the members of the INTEGRATE Consortium for helpful discussions. The INTEGRATE Consortium investigators in the United Kingdom are Sarah J O'Brien (principal investigator), Frederick J Bolton, Rob M Christley, Helen E Clough, Nigel A Cunliffe, Susan Dawson, Elizabeth Deja, Ann E Durie, Sam Haldenby, Neil Hall, Christiane Hertz-Fowler, Debbie Howarth, Lirije Hyseni, Miren Iturriza-Gomara, Kathryn Jackson, Lucy Jones, Trevor Jones, K Marie McIntyre, Charlotte Nelson, Lois Orton, Jane A Pulman, Alan D Radford, Danielle Reaves, Helen K Ruddock, Darlene A Snape, Debbi Stanistreet, Tamara Thiele, Maya Wardeh, David Williams, and Craig Winstanley (University of Liverpool), Kate Dodd (NIHR Clinical Research Network: North West Coast), Peter J Diggle, Alison C Hale, Barry S Rowlingson (Lancaster University), Jim Anson, Caroline E Corless, Viki Owen (Royal Liverpool and Broadgreen University Hospitals NHS Trust), Malcolm Bennett (University of Nottingham), Lorraine Bolton, John Cheesbrough, Katherine Gray, David Orr, Lorna Wilson (Lancashire Teaching Hospitals NHS Foundation 
Trust), Andrew R Dodgson, Ashley McEwan (Manchester University NHS Foundation Trust), Paul Cleary, Alex J Elliot, Ken H Lamden, Lorraine Lighton, Catherine M McCann, Matthieu Pegorie, Nicola Schinaia, Anjila Shah, Gillian E Smith, Roberto Vivancos, Bernard Wood (PHE), Rikesh Bhatt, Dyfrig A Hughes (Bangor University), Rob Davies (APHA); Simon de Lusignan, Filipa Ferreira, Mariya Hriskova, Sam O’Sullivan, Stacy Shinneman and Ivelina Yonova (University of Surrey/Royal College of General Practitioners).

The datasets generated during this study are not publicly available because of the issues of data confidentiality and patient identifiable information. Data are, however, available from the authors on reasonable request and with appropriate ethical permissions.

\section{Conflicts of Interest}

None declared.

\section{References}

1. Tam CC, Rodrigues LC, Viviani L, Dodds JP, Evans MR, Hunter PR, IID2 Study Executive Committee. Longitudinal study of infectious intestinal disease in the UK (IID2 study): incidence in the community and presenting to general practice. Gut 2012 Jan;61(1):69-77 [FREE Full text] [doi: 10.1136/gut.2011.238386] [Medline: 21708822]

2. The University of Liverpool. 2017. Small Animal Veterinary Surveillance Network (SAVSNET) URL: https://www. liverpool.ac.uk/savsnet/ [accessed 2017-08-21] [WebCite Cache ID 6ssrFmYLX]

3. Royal College of General Practitioners. 2019. RCGP Research and Surveillance Centre URL: https://www.rcgp.org.uk/ clinical-and-research/our-programmes/research-and-surveillance-centre.aspx [accessed 2019-08-07]

4. Mengelle C, Mansuy JM, Prere MF, Grouteau E, Claudet I, Kamar N, et al. Simultaneous detection of gastrointestinal pathogens with a multiplex Luminex-based molecular assay in stool samples from diarrhoeic patients. Clin Microbiol Infect 2013 Oct;19(10):E458-E465 [FREE Full text] [doi: 10.1111/1469-0691.12255] [Medline: 23714194]

5. Assets: How They Work. 2013. Microbiology Services: Preparedness and Response London 2012 Olympic and Paralympic Games URL: https://www.gov.uk/government/uploads/system/uploads/attachment data/file/398956/2.

7 Microbiology Services London 2012 report.pdf [accessed 2019-08-07] [WebCite Cache ID 6ssup9AUt]

6. Kind P, Dolan P, Gudex C, Williams A. Variations in population health status: results from a United Kingdom national questionnaire survey. Br Med J 1998 Mar 7;316(7133):736-741 [FREE Full text] [doi: 10.1136/bmj.316.7133.736] [Medline: 9529408]

7. Ritchie J, Lewis J, Elam G. Designing and selecting samples. In: Ritchie J, Lewis J, editors. Qualitative Research Practice: A Guide for Social Science Students and Researchers. Thousand Oaks, CA: Sage Publications; 2005:77-108.

8. Public Health England. 2017. Select Survey URL: https://surveys.phe.org.uk/ [accessed 2019-08-07]

9. McColl E, Jacoby A, Thomas L, Soutter J, Bamford C, Steen N, et al. Design and use of questionnaires: a review of best practice applicable to surveys of health service staff and patients. Health Technol Assess 2001;5(31):1-256 [FREE Full text] [doi: 10.3310/hta5310] [Medline: 11809125$]$

10. Brix A, Diggle PJ. Spatiotemporal prediction for log-Gaussian Cox processes. J Royal Stat Soc 2001 Nov;63(4):823-841. [doi: 10.1111/1467-9868.00315]

\section{Abbreviations}

APHA: Animal and Plant Health Agency

CAG: Confidential Advisory Group

GI: gastrointestinal

GP: general practitioner

HPRU: Health Protection Research Unit

ICER: incremental cost-effectiveness ratios

NHS: National Health Service

NICE: National Institute for Health and Care Excellence

NIHR: National Institute for Health Research

PCR: polymerase chain reaction

PHE: Public Health England

QALY: quality-adjusted life-year

RCGP RSC NMN: Royal College of General Practitioners' Research and Surveillance Centre National Monitoring Network

xTAG GPP: Luminex xTAG Gastrointestinal Pathogen Panel 
Edited by G Eysenbach; submitted 07.03.19; peer-reviewed by S Pesälä, YS Lo; comments to author 26.05.19; revised version received 15.07.19; accepted 16.07.19; published 26.09.19

Please cite as:

McIntyre KM, Bolton FJ, Christley RM, Cleary P, Deja E, Durie AE, Diggle PJ, Hughes DA, de Lusignan S, Orton L, Radford AD, Elliot AJ, Smith GE, Snape DA, Stanistreet D, Vivancos R, Winstanley C, O'Brien SJ

A Fully Integrated Real-Time Detection, Diagnosis, and Control of Community Diarrheal Disease Clusters and Outbreaks (the INTEGRATE Project): Protocol for an Enhanced Surveillance System

JMIR Res Protoc 2019;8(9):e13941

URL: https://www.researchprotocols.org/2019/9/e13941

doi: $10.2196 / 13941$

PMID: 31573952

CKirsty Marie McIntyre, Frederick J Bolton, Rob M Christley, Paul Cleary, Elizabeth Deja, Ann E Durie, Peter J Diggle, Dyfrig A Hughes, Simon de Lusignan, Lois Orton, Alan D Radford, Alex J Elliot, Gillian E Smith, Darlene A Snape, Debbi Stanistreet, Roberto Vivancos, Craig Winstanley, Sarah J O'Brien. Originally published in JMIR Research Protocols (http://www.researchprotocols.org), 26.09.2019 This is an open-access article distributed under the terms of the Creative Commons Attribution License (https://creativecommons.org/licenses/by/4.0/), which permits unrestricted use, distribution, and reproduction in any medium, provided the original work, first published in JMIR Research Protocols, is properly cited. The complete bibliographic information, a link to the original publication on http://www.researchprotocols.org, as well as this copyright and license information must be included. 